\title{
Los 35 años del Programa de Neumología de la Pontificia Universidad Javeriana en el Hospital Universitario San Ignacio
}

La Unidad de Neumología del Hospital Universitario San Ignacio celebra 35 años de su creación y seria inaudito recordar la historia del programa de Neumología sin hablar del Doctor Dario Maldonado, su fundador y director durante 15 años hasta 1992.

La Unidad de Neumología nace en la década de los 70. El Doctor Maldonado regresa a Colombia posterior a su formación en Medicina Interna y Neumología en el Hospital Monte Sinaí de Chicago y el Hospital de Veteranos de Milwaukee, donde aprende una Neumología que trasciende el campo de la Tisiología, en el cual la fisiología, la fisiopatología, las pruebas de función pulmonar y los avances en radiología toman un papel importante dentro de la formación de los neumólogos y en el ejercicio de la misma. Se vincula al Departamento de Medicina Interna del Hospital San Ignacio y a la Facultad de Medicina de la Pontificia Universidad Javeriana, y en su visión de Maestro, le da vida a la Unidad de Neumología del Hospital.

Ésta se ubica inicialmente en dos habitaciones del cuarto piso del Hospital, dentro del servicio de Cirugía, pues en el quinto piso, donde se ubicaba Medicina interna, no había espacio. Un grupo diverso de personas con interés y entusiasmo por la Neumología, bajo la dirección del Doctor Maldonado comienza a darle vida a la Unidad: Beatriz Diazgranados se vincula como técnica del laboratorio de función pulmonar para la realización de los estudios de espirometría; Stella de Heredia y Clara Inés Garcia, junto con Beatriz Almaza se convierten en las pioneras de la terapia respiratoria en el Hospital y en la ciudad; la enfermera Sylvia Baptiste de Arroyo se vincula a la Unidad para apoyar la tarea de coordinación y organización de la misma, siempre orientada al cuidado y bienestar de los pacientes; un grupo de auxiliares, entre ellas Maria Sacramento Esguerra, se entrena para realizar muchas de las tareas diarias.

La Unidad entra en funcionamiento con muy pocos equipos: un espirómetro portátil y un espirómetro de tanque, el famoso "Tissot"; luego se adquiere la máquina de gases arteriales, manejada por los residentes de Medicina interna, y en la medida en que su uso se hace más frecuente y necesario, el grupo de auxiliares entrenadas apoya el manejo de ésta y la realización de las pruebas.

Más tarde, la Unidad se traslada al quinto piso del Hospital y comienza a consolidarse; el objetivo trazado por el Doctor Maldonado desde su inicio comienza a cumplirse: tener una Unidad de Neumología con fortalezas en el área de fisiología y fisiopatología que permitiera hacer una mejor aproximación a la clínica de los pacientes y que fuera un poco diferente a las tradicionales unidades de tisiología de la época.

El Doctor Jorge Restrepo Molina viene de Antioquia y se vincula a la Unidad para fortalecer la docencia. La tarea clínica era apoyada por el servicio de Radiología con los doctores Gastón Morillo y Humberto Varón. Junto con ellos y los residentes de Medicina interna comienzan a desarrollarse actividades académicas como la revisión de casos, la revista docente, la reunión de Radiología y la lectura de las pruebas de función pulmonar.

El Doctor Maldonado, un gran clínico, un excelente neumólogo, un verdadero médico de sus pacientes, pero ante todo un Maestro, crea el programa de posgrado de Neumología, el cual inicia con el Doctor Antonio Acevedo como su primer fellow. Este fue el primer programa de subespecialidad que tuvo la Facultad de Medicina de la Universidad Javeriana.

A lo largo de los años, especialmente en la década de los ochenta, la Unidad de Neumología crece bajo la dirección del Doctor Maldonado; el estudio de la fisiología pulmonar avanza, se desarrollan e implementan nuevas pruebas y estudios de la función pulmonar algunos de ellos con equipos y programas diseñados dentro de la misma Unidad, como las primeras pruebas de ejercicio pulmonar usando el neumotacógrafo, la caja mezcladora, 
las bolsas de Douglas, los analizadores de gases y programas de Excel para realizar los cálculos, la prueba de reinhalación de $\mathrm{CO}_{2}$ con circuitos manuales y mezclas preparadas usando el capnógrafo adquirido en la época, la prueba de metacolina, rudimentarios polisomnogramas con el uso de los poligrafos del Departamento de Ciencias Fisiológicas, y el monitoreo manual de otras variables. Simultáneamente se desarrollan otras áreas como terapia respiratoria y complementos como los "ejercicios respiratorios" que serian la base para la construcción del programa de rehabilitación pulmonar.

La Unidad comienza a ser un sitio de remisión de pacientes complejos, lo que lleva paralelamente al desarrollo de otras áreas como: el manejo del paciente crítico en la Unidad de Cuidado Intensivo, la ventilación mecánica pasando de los ventiladores de presión a los ventiladores de volumen y la hemodinamia con el uso del cateterismo derecho para el estudio de la hipertensión pulmonar.

Todos estos progresos han sido parte de nuestra historia y de lo que hoy somos; fueron momentos muy significativos, enmarcados por años de trabajo y esfuerzo para tener cada dia una Unidad de Neumologia mejor y más avanzada, y para contar con un programa más completo que formara excelentes neumólogos, siempre con el objetivo principal de servir a los pacientes, tal como lo concibió su fundador en su visión de la Unidad del futuro.

Desde la década de los noventa hasta hoy, la Unidad ha crecido, se ha modernizado, cuenta con equipos de última tecnología y se ha consolidado en diferentes áreas como: clínicas de asma, EPOC, hipertensión pulmonar, sueño, tuberculosis, vía aérea, broncoscopia intervencionista y rehabilitación pulmonar. El Programa de Neumología ha formado 37 neumólogos y ha contribuido con la formación de especialistas en áreas como Medicina interna, Anestesia, Medicina familiar, Geriatría y Cirugía del tórax.

La Unidad de Neumología y el programa de posgrado tendrán siempre una deuda de gratitud con su gestor y fundador. Quienes hemos tenido el privilegio de ser alumnos del Doctor Darío Maldonado conocemos el verdadero significado de la palabra "Maestro", pues de él aprendimos la importancia de ser buenos clínicos, partiendo de la fisiología y la fisiopatología en el abordaje del paciente, aprendimos a ejercer el arte de la medicina con excelencia, con compromiso, con entrega, con honestidad..., pero sobre todo aprendimos a pensar primero en el paciente y a poner todo nuestro conocimiento y capacidades al servicio de éste.

Celebrar 35 años de existencia significa, así mismo, rendir un tributo de gratitud al Doctor Darío Maldonado y a todas las personas que han sido parte de la historia de la Unidad, que han aportado con su trabajo y sus ideas al crecimiento de la misma.

Nuestro reto será seguir formando nuevas generaciones de neumólogos con excelencia académica, con interés por la investigación y la docencia, pero ante todo con un gran sentido humano y compromiso con sus pacientes; ellos, a su vez, tendrán la responsabilidad de construir un nuevo capítulo de la historia, lleno de logros y desarrollos en el campo de la Neumología.

Mary Bermúdez Gómez, MD., MSc. Internista, Neumóloga, Decana Académica, Facultad de Medicina, Pontificia Universidad Javeriana Bogotá, Colombia. E-mail: mbermude@javeriana.edu.co 